\title{
USING A TRAIL CAMERA FOR RECORDING PLANT-INSECT INTERACTIONS WITH VENUS FLYTRAP AS AN EXAMPLE
}

\author{
Stephen E. Williams • Emeritus Professor $・$ Lebanon Valley College $・$ Annville $・$ Pennsylvania \\ 17003・USA・williams@1vc.edu \\ BILL SCHOLL・Liberty County・Florida 32321・USA・scholljunk@gmail.com
}

\begin{abstract}
Trail cameras can be used for the study of the interaction between plants and insects if they have a close-up lens and the capability of doing time-lapse photographs or videos. Typical trail cameras are mounted vertically, often on unstable camera stands. We have used the Bushnell NatureView HD Cam Model\# 119740 and RC Williams Co. TopView Quick-Mount camera-stand to study the behavior of insects interacting with Venus flytraps (Dionaea muscipula). The combination allows photographs and videos to be made of insects and plants from above, with the camera held in a position that allows the plants to be observed from the exact same position for months at a time. Every five minutes a photograph and a one-minute video are recorded. We have developed methods of searching through this data for important events that involve producing "slide shows" of the photographs overlain by a grid, that can be quickly scanned for events of interest. Video of these events near the time of the sequentially numbered photograph can then be viewed. The slides and videos can also be sampled at intervals for more gradual changes.
\end{abstract}

\section{Introduction}

Trail Cameras, also called Camera traps, are commonly used for wildlife photography. Mammologists and ornithologists who study animal behavior have adopted versions of these cameras with close up lenses to observe small birds and mammals (Randler \& Kalb 2018; Rovero et al. 2013). In these studies, a Passive Infra-Red (PIR) motion sensor is used to trigger the camera when objects warmer than the background move within the range of the sensor. Plants, as well as insects and other arthropods, are not warmer than their background so PIR sensors will not work. However, a camera capable of taking time-lapse photographs can sample activity by arthropods interacting with plants and also follow changes in the plants themselves with time. Camera traps are weatherproof and are capable of recording images during both dark and light periods. A range of close-up lenses allows them to record plants and animals of various sizes as they interact. The major problem is the large volume of data produced where little of interest is occurring. This creates problems with locating photographs and videos with relevant information within a vast sea of data.

We have been using a Bushnell NatureView HD Cam Model \#119740 with a $460 \mathrm{~mm}$ close up lens to photograph insect and spider interactions with Venus flytrap leaves. The NatureView camera has the capability of making time-lapse photographs and videos. We are using it to take photographs and videos at 5-minute intervals and have developed ways to scan the data for activity so that all data need not be viewed in order to find information of interest.

Another problem we have had is that camera traps and their various camera mounts are designed to be upright in less than stable positions, while for many studies of plants and arthropods, having the camera face downward is the most effective position, and stability is important in any serious photography. We have cooperated with R.C. Williams Co. in the development of the TopView Quick-Mount, a very stable camera mount that points the camera downward. 
The object of this paper is to describe the NatureView camera, and the TopView Quick-Mount as used for studying insect-plant interactions. We will cover their features and their limitations and show examples of their performance.

\section{Capabilities}

The Bushnell NatureView HD Cam Model \#119740 and RC Williams Co. TopView Quick-Mount (Fig. 1) can be used to record the development of plants over extended periods of time (Fig. 2) and record plant insect interactions (Fig. 3 \& 4, Williams \& Scholl 2021). In daylight the HD videos, that record 30 frames per second, can be viewed frame by frame to measure rapid movements (Fig. 4). The videos and photographs also allow the measurement of the frequency of events, such as Dionaea trap closures, and the times of day during which they occur. The camera allows plants and the animals interacting with them to be observed at all hours of the day, over extended periods of time, in all types of weather, without human presence.

\section{Equipment}

The camera: The Bushnell NatureView HD Cam Model \#119740 camera (Fig. 1) can record photographs (JPG files) and also video (AVI files) of 5 seconds to 60 seconds in length. It has a camera mode where only photographs are recorded, a video mode where only video is recorded and a hybrid mode where 1 to 3 photographs are followed after about a second by a video.

The camera is designed to be triggered by a Passive Infra-Red (PIR) motion sensor which is not useful for recording insect plant inter-

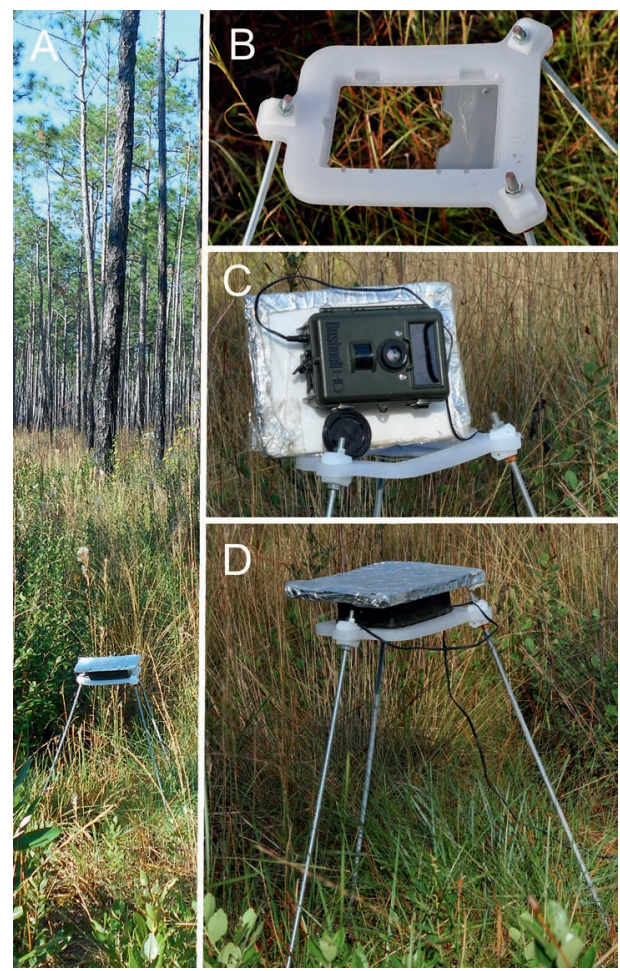

Figure 1: (A) The camera and camera stand at site-02 in a Florida coastal savanna. (B) The TopView Quick-Mount camera stand showing the neutral density filter for the IR flash. (C) The NatureView camera with its $460 \mathrm{~mm}$ close up lens. (D) Closer shot of the camera and stand showing the foam and foil covering that prevents overheating in direct sunlight. actions, but it also has a feature called Field Scan that, in combination with a feature called Interval, can be set to create time-lapse photographs at intervals of 1, 5, 15,30, and $60 \mathrm{~min}$ and videos at intervals of 5, 15, 30, and $60 \mathrm{~min}$. A hybrid mode scan also can produce a photograph followed by a video at intervals of $5,15,30$, and $60 \mathrm{~min}$.

We use a time lapse interval of 5 minutes and a hybrid mode to record a series of $14 \mathrm{M}$ Pixel photographs, each of which is followed by a $1920 \times 1080$ resolution HD video clip 1.0 min in length. A 128 GB SD card will store over 3.5 days of data. 
With a $460 \mathrm{~mm}$ close up lens the NatureView camera covers a $25 \mathrm{~cm} \times 35 \mathrm{~cm}$ field of view at a distance of $46 \mathrm{~cm}$ in photographs and $15 \mathrm{~cm} \times 24.5 \mathrm{~cm}$ in videos. Objects between $39 \mathrm{~cm}$ and $57 \mathrm{~cm}$ from the lens are in focus.

The camera has an IR flash system that allows nighttime photographs and videos. Eight of the 40 IR LEDs are used when the flash is set for "Low". This is still far too intense for close up photography. A holder has been added to the TopView Quick-Mount that allows stainless steel sieve screening to be used as a neutral density filter that lowers the IR intensity to usable levels (Fig. 1). Nighttime photographs and videos are, at their worst something like an ultrasound image, and at their best like a grainy black and white photograph (Fig. 5), but they allow a view of behavior that is otherwise impossible to achieve.

The camera mount: Figure 1 shows the Bushnell NatureView camera mounted on an RC Williams Co. TopView Quick-Mount. The camera can be removed to change SD cards and batteries, and to reset the clock. After maintenance operations, the camera can be placed back into the exact position it was previously in. The three legs are 5/16-inch galva-
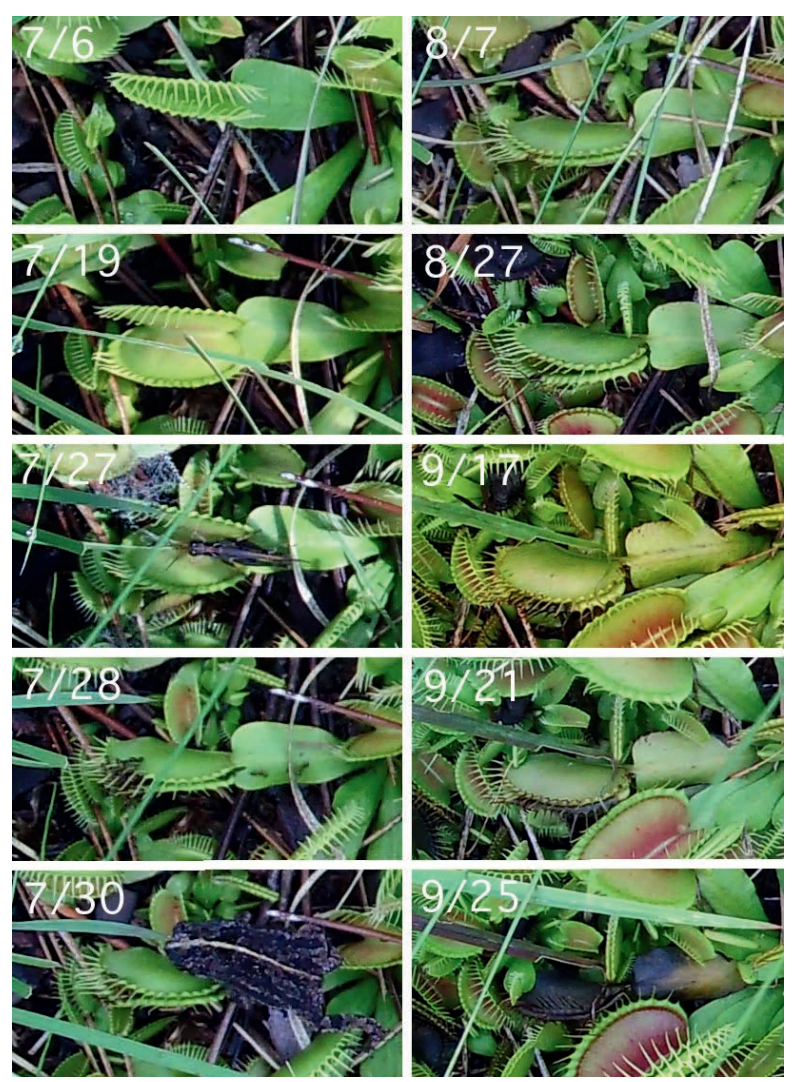

Figure 2: This sequence of photographs follows a single Dionaea leaf from July 6 when its trap was not fully open until September 25 when it was dead. The camera stand was adjusted slightly between 8/27 and 9/17 to improve the focus, otherwise all shots were made without changing the camera's position. On 7/27 the trap captured a grasshopper large enough that the lobes of the trap could not come completely together. By the next day (7/28) ants were visiting the trap and feeding on the partly digested grasshopper. The ants attracted an Oak Toad (Anaxyrus quercicus) that fed on them for a while. The trap resealed (8/7) and then partly opened (8/27) but was never functional again except as a photosynthetic organ. The trap began to die about 9/21 and was dead by 9/25.

nized threaded rod cut to the length required. With the legs pushed into the ground, the platform is stable enough that we have taken photographs of the exact same area over several months with no noticeable deviation in the field of view covered by the camera. The camera mount is HDPE machined to exactly hold the camera so that no strap or fasteners are needed to hold it in place. 

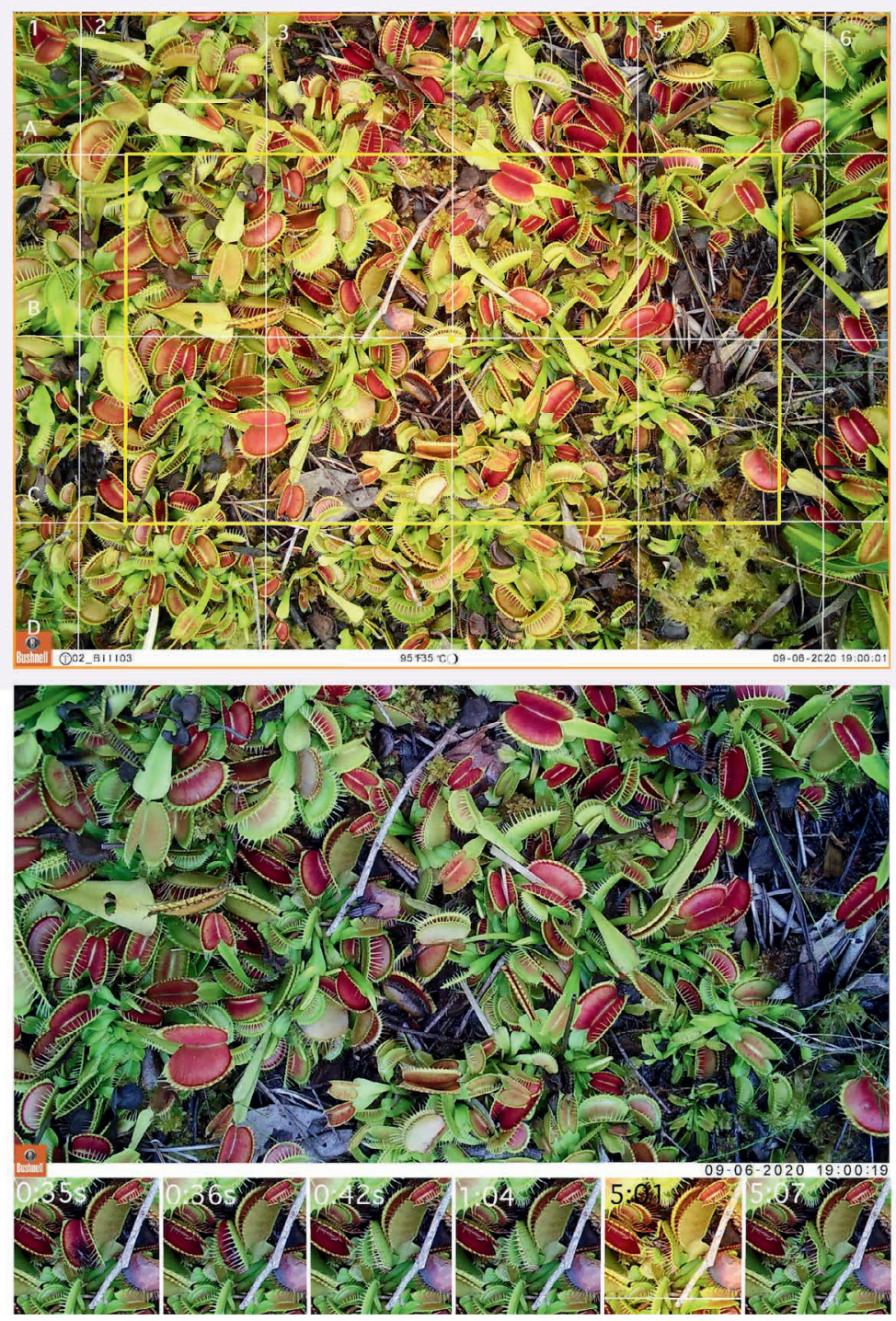

Figure 3: The top photograph (JPG) has been overlain with a grid and had a white border added. The yellow rectangle in the center of the top photo delineates the area that will be visible in the video. An individual frame from the video (AVI), recorded just after the photograph, is pictured below the top photograph. It is from 18 seconds after the first frame. Note that there is a weevil on the stick that does not appear in the photograph above. This video was located by scanning through a "slide show" of photographs similar to the top one and locating a trap that closed between the photo pictured and the following photo taken at 19:05:01 part of which is pictured in the 5th square at the bottom of the illustration. The trap, in grid section B3 of the photograph at the top of the page, snapped shut after the weevil entered it. Sections from the frames of the video show the weevil entering the trap and being captured. Four minutes after the capture video the weevil forces its way out of the trap as can be seen in the last two frames in the bottom series. 


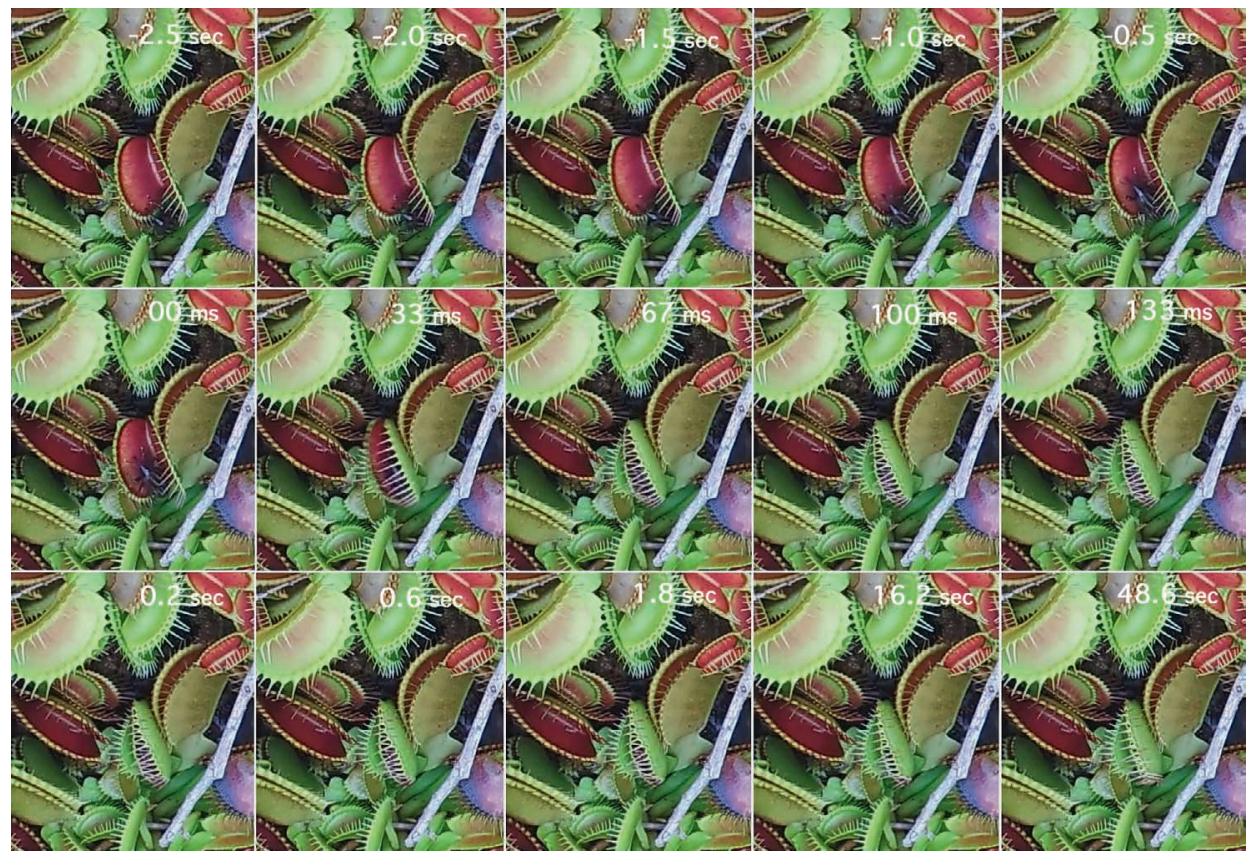

Figure 4: Closure of the trap triggered by the weevil in Figure 3. Individual frames of the video were recorded at 30 frames a second, $33 \mathrm{~ms}$ (0.033 seconds) per frame. The beginning of trap closure occurred between the first frame in the second row (00 ms) and the next frame $(33 \mathrm{~ms})$. The rapid phase of trap closure was complete by $67 \mathrm{~ms}$. A slower phase of closing occurred between $67 \mathrm{~ms}$ and 48.6 seconds when the trap was fully closed. Time zero is the last frame of the video where the trap was fully open. Frames before time zero are listed as negative numbers. The temperature at closure was measured by the camera as $35^{\circ} \mathrm{C}$. This figure was composed by Siegfried Hartmeyer using EDIUS X PRO video editing software.

Shadows of the camera and mount are evident in photographs and videos taken on clear sunny days. On hot days when the camera is in direct sunlight the horizontally mounted camera is subject to overheating. The overheating problem was solved by placing an aluminum foil covered Starfoam board over the camera to protect it (Fig. 1).

Data storage: Bushnell recommends using SDHC cards of up to 32 GB capacity, but we have used $64 \mathrm{~GB}$ and $128 \mathrm{~GB}$ cards without problems. Larger $256 \mathrm{~GB}$ cards will not work. The camera formats SD cards with FAT 16 format, which is readable by both MacOS and Windows operating systems. A $128 \mathrm{~GB}$ card holds about 3.5 days of our data and a $64 \mathrm{~GB}$ card holds about 1.75 days of data. Shooting lower resolution video and photographs will allow far more photographs and video clips to be stored on a card.

Power: Recording photographs and video in hybrid mode at the resolution we use will use up a set of 12 alkaline batteries in recording the 42 hours of data that will fill a single $64 \mathrm{~GB}$ card. The camera can be powered externally using an AC Power Adapter, allowing $128 \mathrm{~GB}$ cards to be used. An AC adapter can be used inside and, in a garden, where power is available. We have used an AC power adapter with a 200-foot extension cord to power a camera at one of our field sites. We have also successfully used three 6-volt, rechargeable 4-AH lead acid batteries in paral- 
lel. AC power adapters and rechargeable batteries are both much less expensive and more environmentally friendly than using AAA cells. However, AAA cells should be kept in the camera to hold the settings when it is not plugged into a power source. The IR flash uses a lot of power. Battery life is greatly extended if the camera is run in daytime mode.

\section{Organizing and storing data}

The data on the SD card is in folders of 999 or fewer files. After data collection our 128 GB SD cards have three folders with alternating JPG and AVI files ordered by file name within a folder. The name is a number like 09110168 where 0911 indicates that the photograph or video was taken on, September 11, and 0168 indicates it is the 168th file in the folder. If a 128 GB SD card is full it will have three folders

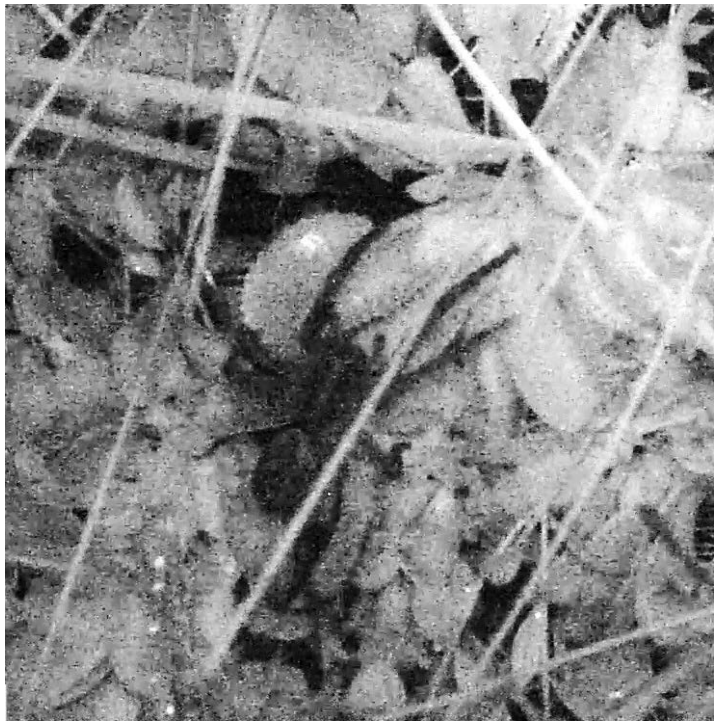

Figure 5: An image of a large spider walking over Dionaea leaves at midnight. It measures approximately $6.5 \mathrm{~cm}$ from the tip of its front leg to the tip of its back leg. The image is typical of those recorded in the dark with the IR flash. Note that the traps are visible. Their closures during the dark period can be detected. in it. The first two folders will have 999 files in them and the third will have 42 files. The files can be sorted by type resulting in AVI files rising to the top of the list. The JPGs can be placed in a separate folder resulting in a folder of JPG files and a folder of AVI files. The folders can be named with the date of the first file they contain followed by AVI or JPG. File 251 JPG will have been taken a second before file 252 AVI. This allows the photographs to be matched with the videos.

We use the application, BatchPhoto, to put a grid on every JPG file in the folder (Fig. 3). This serves like the grid on a map and allows the location of traps within the photograph. It also outlines in yellow the area that will appear in the videos.

The JPG files with the grid can be added to an Apple Photos or Windows Photo library and a slide show created. The slide show can be quickly scrolled through while watching one sector of the grid while looking for changes such as trap closures, the appearance of animals, etc. The file number, date, and time when these events occur can then be noted. After scrolling through the slide show making notes on all sectors, the videos where the event occurred can be viewed. Since one minute of every five is recorded on video there is a $20 \%$ chance that the event, such as a trap closure was captured on video. The events can also be tallied and their frequency of occurrence can be determined, as can the distribution of times at which the events occurred.

In the light video is recorded at 30 frames per second. The sequence of video frames taken every $33 \mathrm{~ms}$ can be used to record the time required for rapid events to occur (Fig. 4).

Once an event, such as the capture of an insect, is recorded the behavior of the trap can be followed and the time of reopening determined. In the extreme, the entire life of a leaf can be followed over a period of many weeks (Fig. 2). 


\section{Conclusion}

While camera traps have been very useful for documenting warm-blooded animals, plants and insects cannot be photographed using PIR triggered cameras. Properly positioned camera traps with time-lapse capability allow photos in all weather, day and night, over extended periods of time. Methods of searching the photographs and videos for activity then allow studies of plant insect interactions. We have successfully observed the interaction between Dionaea traps and visiting insects and spiders and between Dionaea flowers and their visitors (Williams \& Scholl 2021).

Even without being able to use its PIR sensors, the camera trap opens up a window allowing a view of the relation of insects and plants. It takes more patience than using a deer camera looking at big game does, but it offers the opportunity to see what no one has seen before.

Acknowledgements: We would like to thank Rick Williams of RC Williams Co. for designing the camera holder that played a crucial roll in this research. We thank Dr. Susan Verhoek of Lebanon Valley College for constructive comments on the research. Figure 4 is by Siegfried Hartmeyer who separated the video stills with EDIUS X PRO video editing software. We also thank Dr. Bruce Means, of Florida State University for identifying the Oak Toad Anaxyrus quercicus pictured in Figure 2.

Disclosure: The authors have no financial interest in either Bushnell Corp. or RC Williams Co.

\section{References}

Randler, C., and Kalb, N. 2018. Distance and size matters: A comparison of six wildlife camera traps and their usefulness for wild birds. Ecology and Evolution 8(14): 7151-7163.

Rovero, F, Zimmermann, F, Berzi, D., and Meek, P. 2013. "Which camera trap type and how many do I need?" A review of camera features and study designs for a range of wildlife research applications. Hystrix. 24(2): 148-156.

Williams, S.E., and Scholl, B. 2021. Pollination of Dionaea muscipula, the Venus Flytrap. Carniv. Pl. Newslett. 50(1): 16-23.

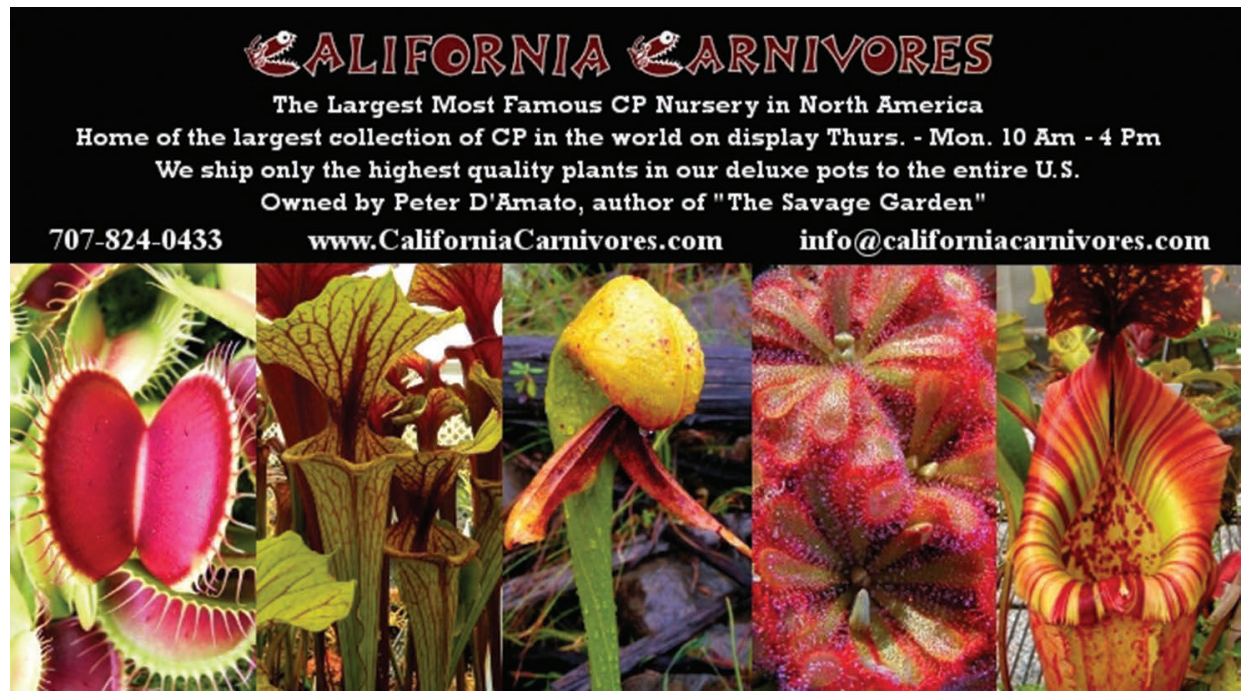

